\title{
ESTUDIO DENDROCLIMÁTICO DE LAS RACHAS SECAS Y HÚMEDAS EN EL SECTOR CENTRAL DE LA DEPRESIÓN DEL EBRO ${ }^{1}$
}

\author{
Miguel Ángel SAZ SÁNCHEZ y José CREUS NOVAU \\ Instituto Pirenaico de Ecología \\ Consejo Superior de Investigaciones Científicas, Jaca (Huesca)
}

\begin{abstract}
Resumen: Con la serie dendroclimática de la precipitación de Pallaruelo de Monegros, observatorio situado en el sector centro-oriental de la depresión del Ebro, se realiza un estudio de las rachas secas y húmedas sucedidas entre los años 1465 y 1989, analizando su frecuencia, duración e intensidad. La caracterización de los años en función de su total pluviométrico ha permitido observar como hechos más destacados la ausencia casi total de secuencias de años categorizados como normales y el comportamiento excepcional de la precipitación en el siglo XX.
\end{abstract}

Palabras clave: Dendroclimatología, Valle del Ebro, sequías, variabilidad pluviométrica.

\begin{abstract}
With the dendroclimatic series of precipitation in Pallaruelo de Monegros weather station (placed in the mid-eastern sector of the Ebro Basin), we study the dry and humid periods between years 1465 and 1989, analysing its frequency, duration and intensity. The characterization of the years according to its total precipitation has allowed us to see as most remarkable facts the almost total lack of sequences of normal years and the exceptional behaviour of the precipitation in XXth century.
\end{abstract}

Key Words: Dendroclimatology, Ebro valley, drought, rainfall variability.

\section{INTRODUCCIÓN}

En cualquier aproximación al clima del centro de la depresión del Ebro es inevitable hacer referencia a la penuria pluviométrica. La sequedad es sin duda el rasgo más destacable de la zona, haciéndose patente en el paisaje y en topónimos que de alguna forma hacen referencia a la escasez de agua, como la Valsalada, Valdespartera, las Polvorosas, Ontinar, Desierto de la Violada o Desierto de Calanda.

${ }^{1}$ Este trabajo forma parte del proyecto de investigación CLI96-1862, financiado por la CICYT. 
Los reducidos totales de precipitación van unidos en este caso a una elevada variabilidad interanual. Frente a años donde las precipitaciones superan ampliamente el valor obtenido para la media del periodo estudiado, se observan otros donde lo recogido no llega ni siquiera a un tercio de esa cantidad. Los promedios se convierten así en valores escasamente significativos, siendo poco habitual la presencia de años que muestren totales cercanos a ese parámetro.

Además de estas dos características, las sequías y los largos periodos secos son otros de los rasgos definitorios del clima del sector central de la depresión del Ebro, como se ha puesto de relieve en muchos de los trabajos publicados (ASCASO et al., 1981; CUADRAT, 1989; CUADRAT, 1991; PÉREZ et al., 1994; CREUS et al., 1995; CREUS 1996; DEL VALLE, 1996).

La presencia de años consecutivos con totales pluviométricos exiguos no constituye un hecho extraordinario en esta zona, sino que bien al contrario se convierte en un fenómeno habitual, con las implicaciones socioeconómicas que ello ha supuesto.

Estos estudios han analizado los datos contenidos en los registros instrumentales, cuyas series, en el mejor de los casos, ofrecen información respecto al comportamiento de la precipitación y la temperatura desde el último tercio del siglo XIX. Pero lo más habitual es que las mediciones, y con ello los datos registrados, no se iniciasen hasta la segunda mitad de la presente centuria, y en algunos casos sólo se han tenido en cuenta los últimos 35-40 años. Aunque una serie de datos climáticos de treinta años, e incluso menos, pueda ser suficiente para obtener parámetros estadísticos significativos, no nos permite extrapolar el comportamiento de las variables hacia siglos anteriores y mucho menos acercarnos a sus límites normales de variabilidad.

La dendroclimatología, referida a las reconstrucciones climáticas que se obtienen a partir del crecimiento anual que, en general, experimentan las especies leñosas en nuestras latitudes, constituye una fuente de información que permite acercarnos al problema que supone la escasa longitud de los registros instrumentales. Nos proporciona información de carácter cuantitativo referida al clima de las últimas centurias, con la limitación que impone la longevidad de los árboles utilizados.

Se consiguen así largos registros de información climática, de una elevada resolución temporal (año a año) que posibilitan una evaluación más precisa de la normalidad o anormalidad del clima y permiten el cálculo de parámetros estadísticos más sólidos y fiables, al proceder de una muestra mucho más amplia (CREUS et a1., 1996). 


\section{ANTECEDENTES DENDROCLIMÁTICOS EN EL VALLE DEL EBRO}

Los primeros estudios dendroclimáticos realizados en el ámbito español datan de mediados de los años setenta (CREUS et al, 1976), pero no fue hasta los años noventa cuando experimentaron el empuje necesario que permitiese la creación de un banco de dendrocronologías con el que realizar reconstrucciones climáticas.

En el Valle del Ebro ya se han publicado algunos estudios sobre variables reconstruidas a partir de la información contenida en las cronologías y posteriormente en la base de datos. En el trabajo de 1976, citado anteriormente, se aportan datos que muestran la alternancia de años con características oceánicas y años de filiación mediterránea, marcando unas alternancias cada vez más cortas y frecuentes. Años más tarde (CREUS, 1991) se analizó el comportamiento de la temperatura de mayo en el Pirineo Oriental entre 1302 y 1989, lo que permitió detectar cambios en la tendencia de la temperatura media primaveral, valorar el descenso térmico correspondiente a la Pequeña Edad del Hielo (inferior a $1^{\circ} \mathrm{C}$ ) y observar el incremento de la variabilidad térmica, en comparación con los siglos anteriores, que se produce a lo largo del siglo XX.

Posteriormente este mismo autor estudió la precipitación y temperatura reconstruidas desde el año 1400 en el observatorio de Pallaruelo de Monegros (CREUS et al.,1996). El mayor número de muestras obtenidas y la aplicación de otros enfoques metodológicos posibilitó la reconstrucción de las dos variables, mostrando como ni la temperatura ni la precipitación han tenido una variación continuada de su tendencia en los últimos seis siglos. Ello no se contradice con la presencia de cortos periodos con tendencia de distinto signo, en los que predominaron condiciones más cálidas o frías, en el caso de las temperaturas, y más secas o húmedas, en el caso de las precipitaciones. Dicho trabajo destaca además la gran variabilidad climática de los siglos XV, XVI y primera mitad del XVII (Pequeña Edad del Hielo) y el nuevo incremento observado en el siglo $X X$.

\section{OBJETIVOS}

En los estudios dendroclimáticos ya citados se ha comprobado que la penuria pluviométrica y la elevada variabilidad interanual, si bien con valores más o menos elevados según los periodos, son situaciones habituales en el sector central de la depresión del Ebro desde hace siglos.

El objetivo que se plantea es comprobar si a lo largo de las últimas centurias la presencia de secuencias de años secos (rachas secas) puede ser otro de los rasgos definitorios de la pluviometría de esta zona, para lo que se analizará la duración e 
intensidad de las principales rachas secas previamente definidas. Tal frecuencia, asociada a un clima muy variable, puede condicionar la normalidad de la precipitación, hasta el punto que los años y grupos de años en que las precipitaciones se aproximan a la media sean precisamente la excepción.

\section{MATERIAL Y METODOLOGÍA}

Los datos utilizados proceden de las reconstrucciones dendroclimáticas realizadas tomando como base el observatorio de Pallaruelo de Monegros, situado en el sector centro-oriental de la depresión del Ebro (CREUS et al., 1996), cuya serie de precipitaciones reconstruida abarca el periodo 1465-1989.

Para clasificar los años según su total pluviométrico y agruparlos en categorías con objeto de delimitar las rachas húmedas y secas, se ha utilizado el método de los quintiles, recomendado por el Instituto Nacional de Meteorología y de uso tradicional en climatología (GRISOLLET et al., 1962; ALBENTOSA, 1975; ALMARZA et al., 1994). esquema:

Este método permite clasificar los años en cinco categorías según el siguiente

1.- Años muy secos: precipitación inferior al valor del primer quintil.

2.- Años secos: precipitación entre el primer y el segundo quintil.

3.- Años normales: precipitación entre el segundo y el tercer quintil.

4.- Años húmedos: precipitación entre el tercer y el cuarto quintil.

5.- Años muy húmedos: precipitación superior al valor del cuarto quintil.

Si bien esta metodología implica que el total de años muy secos y secos será de un $40 \%$ y que en el mismo porcentaje van a estar los clasificados como muy húmedos y húmedos, se ha considerado que para detectar rachas de años consecutivos del mismo signo ofrece los mejores resultados.

Otros métodos, como el establecido en 1986 por la Organización Meteorológica Mundial, que considera que hay sequía en una región cuando la precipitación anual es inferior al $60 \%$ de la normal durante más de dos años consecutivos o el de calificar los años según se desvíen sus totales en más o menos un número determinado de 
desviaciones estándar respecto al promedio de la serie, no han sido considerados. El primero porque no permite clasificar los años que no puedan ser categorizados como secos, imposibilitando de esta forma la detección de años normales y húmedos así como secuencias de éstos. El segundo sí permite hacer una clasificación de los años en las tres categorías citadas, pero el criterio empleado es demasiado exigente y sólo detecta los años caracterizados por ser extraordinariamente secos o húmedos, dificultando una adecuada percepción de secuencias de años del mismo signo.

\section{ANÁLISIS DE LOS RESULTADOS}

Enla Tabla 1 aparecen algunos de los estadísticos más significativos obtenidos a partir de la ya citada serie de precipitaciones reconstruida. La aplicación del método de los quintiles permite distinguir cinco grupos de años según su total de precipitación:

$1^{\circ}$.- Años muy secos: precipitaciones inferiores a $363,55 \mathrm{~mm}$.

$2^{\circ}$.- Años secos: precipitaciones entre 363,55 y $406,2 \mathrm{~mm}$.

$3^{\circ}$.- Años normales: precipitaciones entre 406,2 y $440,2 \mathrm{~mm}$.

$4^{\circ}$.- Años húmedos: precipitaciones entre 440,2 y 490,4 mm.

$5^{\circ}$.- Años muy húmedos: precipitaciones superiores a 490,4 mm.

Estos resultados se han trasladado a la Figura 1, donde con objeto de facilitar la percepción de rachas húmedas o secas aparecen bajo la denominación genérica de secos los años clasificados como secos o muy secos, y bajo la de húmedos los muy húmedos o húmedos.

a) Duración de las rachas secas y rachas húmedas.

En la Figura 1 destaca la presencia a lo largo de los 525 años de la serie estudiada de un buen número de rachas secas y rachas húmedas (su número y duración se contabilizan en el Cuadro 2). Aunque el total de unas y otras es bastante parecido, las que han tenido una duración mayor son dos de tipo seco, ocurridas en los siglos XV y XX. La más larga duró diez años y se desarrolló entre 1980 y 1989, seguida de otra de nueve años ocurrida entre 1488 y 1496. Otra secuencia seca larga, esta vez de seis años, también tuvo lugar a lo largo del siglo XX, entre 1944 y 1949. 
Tabla 1.- Estadísticas de los valores de precipitación anual en Pallaruelo de Monegros (1465-1989).

\begin{tabular}{|c|c|c|c|c|c|c|c|}
\hline & $\begin{array}{l}1465- \\
1500\end{array}$ & $\begin{array}{c}1501- \\
1600\end{array}$ & $\begin{array}{c}1601- \\
1700\end{array}$ & $\begin{array}{c}1701- \\
1800\end{array}$ & $\begin{array}{c}1801- \\
1900\end{array}$ & $\begin{array}{c}1901- \\
1989\end{array}$ & $\begin{array}{c}1465- \\
1989\end{array}$ \\
\hline Media & 394,5 & 429,8 & 434,7 & $\overline{423,4}$ & 422,7 & 425,5 & 425 \\
\hline Mediana & 413,5 & 423,6 & 429,7 & 425,5 & 427,9 & 426,4 & 426,3 \\
\hline Varianza & 12204,9 & 9936,6 & 6246,1 & 4981,3 & 2789,8 & 6465,9 & 6514,4 \\
\hline Desv. St. & 110,5 & 99,7 & 79 & 70,6 & 52,8 & 80,4 & 80,7 \\
\hline Mínimo & 131,9 & 190,7 & 283 & 271,5 & 284,6 & 258,4 & 131,9 \\
\hline Máximo & 596,2 & 698,4 & 641,2 & 556,7 & 527,6 & 741 & 741 \\
\hline Coef. var. & 28 & 23,2 & 18,2 & 16,7 & 12,5 & 18,9 & 19 \\
\hline Quintil $1^{\circ}$ & 306,7 & 358,8 & 371 & 365,4 & 385 & 363,7 & 363,5 \\
\hline Quintil $2^{\circ}$ & 372,5 & 406,6 & 413,8 & 400,6 & 412,6 & 402,5 & 406,2 \\
\hline Quintil $3^{\circ}$ & 435,4 & 435,8 & 451,9 & 444,7 & 437,8 & 440,8 & 440,2 \\
\hline Quintil $4^{\circ}$ & 489 & 509,5 & 496,3 & 500,1 & 468,1 & 491,3 & 490,4 \\
\hline
\end{tabular}

Fuente: Creus, J., Fernández, A. y Manrique, E., (1996). Modificado.

Tabla 2 .- Rachas secas y húmedas en el observatorio de Pallaruelo de Monegros (1465-1989): número y duración.

\begin{tabular}{|c|c|c|c|c|c|c|c|c|c|c|c|c|c|c|}
\hline & \multicolumn{2}{|c|}{$1465-1500$} & \multicolumn{2}{|c|}{$1501-1600$} & \multicolumn{2}{|c|}{$1601-1700$} & \multicolumn{2}{|c|}{$1701-1800$} & \multicolumn{2}{|c|}{$1801-1900$} & \multicolumn{2}{|c|}{$1901-1989$} & \multicolumn{2}{|c|}{$1465-1989$} \\
\hline & secas & húm & secas & húm & secas & húm & secas & lúm & secas & húm & secas & húm & secas & hím \\
\hline 1 año & 5 & 5 & 13 & 15 & 13 & 8 & 5 & 9 & 14 & 14 & 9 & $\overline{9}$ & 59 & 60 \\
\hline 2 años & - & 1 & 3 & 4 & 6 & 6 & 9 & 5 & 9 & 5 & 5 & 2 & 32 & 23 \\
\hline 3 años & $\overrightarrow{-}$ & 2 & 2 & 1 & 1 & 1 & 2 & 2 & - & 1 & 1 & 1 & 6 & 8 \\
\hline 4 años & 1 & - & 1 & 2 & 2 & 2 & 1 & 1 & 1 & 1 & - & 2 & 6 & 8 \\
\hline 5 años & - & - & 2 & 1 & - & 1 & 2 & - & - & 1 & - & - & 4 & 3 \\
\hline 6 años & - & - & - & - & - & - & - & 1 & - & - & 1 & 2 & 1 & 3 \\
\hline 7 años & - & - & - & - & - & - & - & 1. & - & - & - & - & - & 1 \\
\hline 8 años & - & - & - & - & - & 1 & - & - & - & - & - & - & - & 1 \\
\hline 9 años & 1 & - & - & - & - & - & - & - & - & - & - & - & 1 & - \\
\hline $\begin{array}{c}10 \\
\text { años }\end{array}$ & - & - & - & - & - & - & - & - & - & - & 1 & - & 1 & - \\
\hline
\end{tabular}

Fuente: Creus, J., Fernández, A. y Manrique, E., (1996). Modificado. 


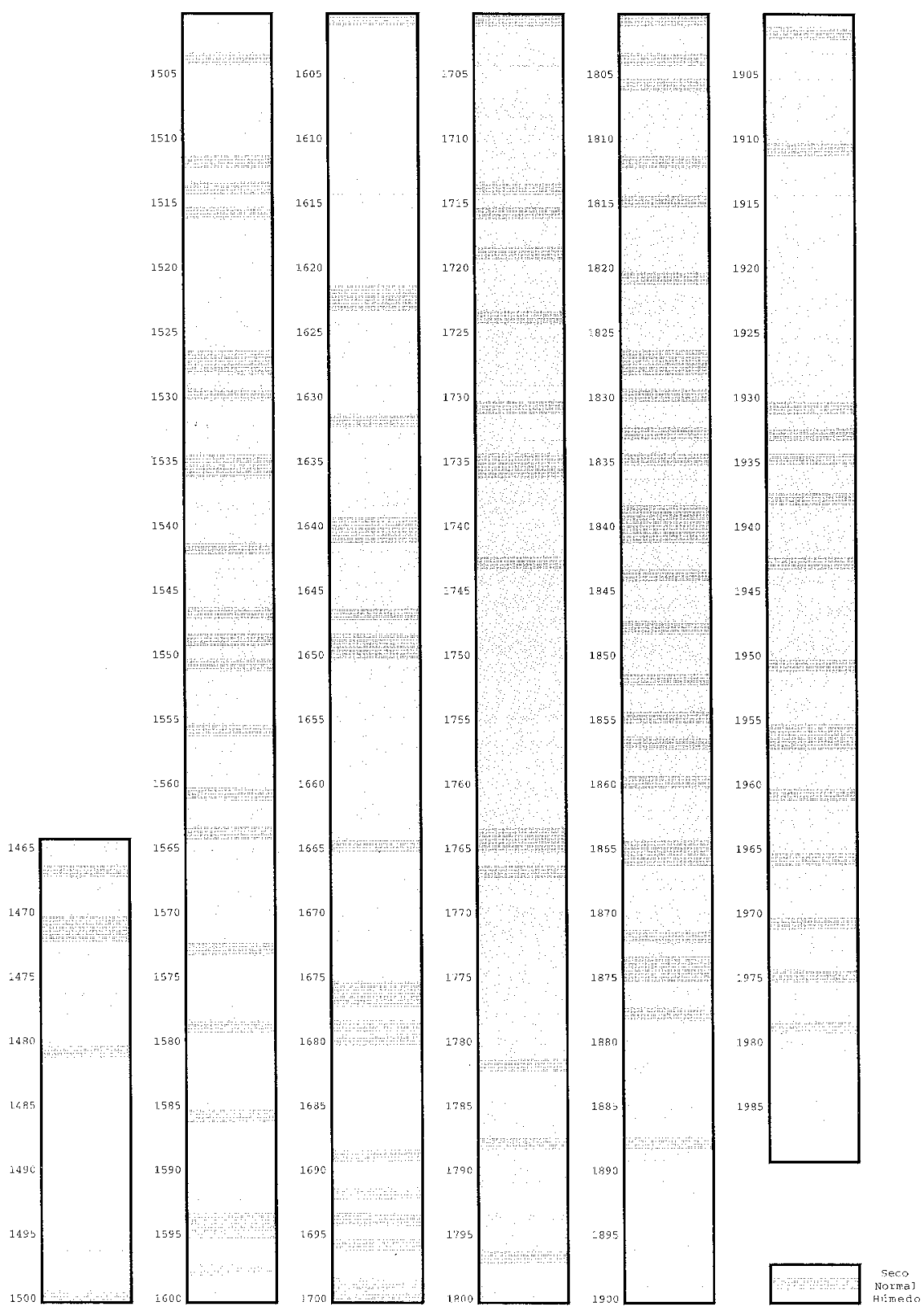

Figura 1.- Caracterización de los años climáticos del observatorio reconstruido de Pallaruelo de Monegros en función de su total pluviométrico (1465-1989). 
Las rachas húmedas más prolongadas parecen tener una duración inferior a las secas, siendo la máxima de 8 años, entre 1607 y 1614. Siguen en longitud otra de siete años, 1701-1777, y tres de seis años: 1758-1763, 1917-1922 y 1925-1930.

El agrupamiento de años del mismo signo formando rachas es más factible entre los años clasificados como secos y húmedos que entre los normales. De éstos últimos, más del $62 \%$ aparecen de forma aislada, mientras que entre los años secos y húmedos este porcentaje desciende, en ambos casos, a tan solo un $28 \%$. Sólo se contabilizan en los 525 años de duración de la serie reconstruida una racha de tres años (1839-1841) y quince rachas de dos años clasificados como normales. Estas cifras son sensiblemente menores a las rachas secas y húmedas de dos y tres años de duración contabilizadas a lo largo de todo el periodo estudiado (ver Tabla 2).

b) Intensidad de las rachas secas y húmedas.

Para valorar la intensidad de las rachas, secas o húmedas, se calculó el promedio de las diferencias respecto a la media del periodo estudiado de cada uno de los años de la racha, dándole el nombre de déficit medio anual para las rachas secas y superávit medio anual para las húmedas. Siendo $P i$ el total de precipitación de cada uno de los años de la racha, $P$ la precipitación media calculada para el periodo 14651989 y $n$ el número total de años de la racha, la fórmula utilizada fue la siguiente.

Del conjunto de rachas detectadas, en la Tabla 3 aparece reflejada la intensidad de aquellas de duración igual o superior a los tres años.

Aunque el número de rachas húmedas de tres o más años de duración contabilizadas en los últimos 525 años es mayor que el de secas, son más las que entre éstas han mostrado intensidades sobresalientes. Estableciendo el umbral de los 100 $\mathrm{mm}$ /año para referirnos a secuencias de intensidad elevada, el número de secas con un déficit medio que supera esa cifra es el doble que el de húmedas: ocho frente a cuatro. Esta mayor fuerza de las rachas secas la confirma además el promedio de los valores obtenidos de intensidad: más de $91 \mathrm{~mm} /$ año en el caso de los déficit frente a $78 \mathrm{~mm}$ /año de los superávit.

En el caso de las rachas secas las que presentan las intensidades más importantes suelen ser además las más largas. La segunda en extensión de la serie (1488-1496) muestra el déficit medio más notable, $154,7 \mathrm{~mm} / \mathrm{año}$, mientras que la de mayor duración (1980-1989) también se presenta como una de las de déficit medio más elevado, superando los $111 \mathrm{~mm} /$ año. Por el contrario, entre las rachas secas que presentan menores déficits (inferiores a $70 \mathrm{~mm} /$ año) no tenemos ninguna de duración superior a los cuatro años. 
Tabla 3.- Intensidad de las principales rachas secas y húmedas del observatorio de Pallaruelo de Monegros (1465-1989).

\begin{tabular}{|c|ccccc|}
\hline $\begin{array}{c}\text { Racha } \\
\text { seca }\end{array}$ & $\begin{array}{c}\mathrm{N}^{\circ} \text { de } \\
\text { años }\end{array}$ & $\begin{array}{c}\text { Déficit } \\
\text { medio }\end{array}$ & $\begin{array}{c}\text { Racha } \\
\text { húmeda }\end{array}$ & $\begin{array}{c}\mathrm{N}^{\mathrm{a}} \mathrm{de} \\
\text { años }\end{array}$ & $\begin{array}{c}\text { Superávit } \\
\text { medio }\end{array}$ \\
\hline $1473-1476$ & 4 & 90,49 & $1468-1470$ & 3 & 55,35 \\
$1488-1496$ & 9 & 154,70 & $1497-1499$ & 3 & 97,41 \\
$1522-1526$ & 5 & 140,68 & $1507-1510$ & 4 & 116,98 \\
$1532-1534$ & 3 & 134,98 & $1517-1521$ & 5 & 169,98 \\
$1544-1546$ & 3 & 111,35 & $1537-1540$ & 4 & 86,20 \\
$1580-1584$ & 5 & 74,66 & $1576-1578$ & 3 & 99,61 \\
$1589-1592$ & 4 & 81,24 & $1602-1605$ & 4 & 75,30 \\
$1615-1617$ & 3 & 79,65 & $1607-1614$ & 8 & 117,98 \\
$1666-1669$ & 4 & 62,24 & $1618-1621$ & 4 & 121,96 \\
$1682-1685$ & 4 & 88,17 & $1628-1630$ & 3 & 90,98 \\
$1725-1729$ & 5 & 109,30 & $1652-1656$ & 5 & 68,76 \\
$1744-1746$ & 3 & 36,88 & $1702-1704$ & 3 & 47,20 \\
$1751-1755$ & 5 & 108,32 & $1710-1712$ & 3 & 79,88 \\
$1768-1770$ & 3 & 150,05 & $1720-1723$ & 4 & 89,28 \\
$1778-1781$ & 4 & 51,72 & $1758-1763$ & 6 & 76,66 \\
$1822-1825$ & 4 & 38,34 & $1771-1777$ & 7 & 42,01 \\
$1944-1949$ & 6 & 73,95 & $1809-1811$ & 3 & 53,35 \\
$1953-1955$ & 3 & 44,92 & $1816-1820$ & 5 & 40,32 \\
$1980-1989$ & 10 & 111,25 & $1883-1886$ & 4 & 59,86 \\
& & & $1907-1910$ & 4 & 66,93 \\
& & & $1912-1914$ & 3 & 66,61 \\
& & & $1917-1922$ & 6 & 55,43 \\
& & & $1925-1930$ & 6 & 46,08 \\
& & & $1939-1942$ & 4 & 56,01 \\
\hline
\end{tabular}

La relación duración-intensidad no aparece de forma tan clara entre las rachas húmedas. La más persistente (1607-1614) aparece asimismo como una de las más intensas, pero en este caso la que le sigue en extensión temporal (1771-1777) es la penúltima en cuanto a su superávit medio anual entre aquéllas de duración igual o superior a los tres años. Tampoco las tres rachas húmedas de seis años detectadas ofrecen unos superávit medios importantes, no rebasando en ningún caso la cantidad de $76 \mathrm{~mm} /$ año.

c) Evolución de la variabilidad pluviométrica y presencia de rachas.

En la Figura 2 se muestra la evolución temporal del valor del coeficiente de variación, medido en intervalos de medio siglo (sus valores aparecen en la Tabla 4). 
Tabla 4.- Valores obtenidos para el coeficiente de variación, precipitación media anual y porcentaje de años secos en intervalos de medio siglo.

\begin{tabular}{|c|c|c|c|}
\hline Intervalo & $\begin{array}{c}\text { Coeficiente de } \\
\text { variación }\end{array}$ & $\begin{array}{c}\text { Precipitación media } \\
\text { anual }\end{array}$ & $\begin{array}{c}\text { Porcentaje de } \\
\text { años secos }\end{array}$ \\
\hline $1465-1500$ & 28,00 & 394,53 & 50 \\
$1501-1550$ & 25,86 & 435,05 & 36 \\
$1551-1600$ & 20,22 & 424,54 & 42 \\
$1601-1650$ & 18,83 & 452,97 & 30 \\
$1651-1700$ & 16,40 & 416,45 & 42 \\
$1701-1750$ & 17,43 & 418,02 & 44 \\
$1751-1800$ & 15,98 & 428,80 & 42 \\
$1801-1850$ & 11,84 & 426,57 & 30 \\
$1851-1900$ & 13,20 & 418,77 & 42 \\
$1901-1950$ & 12,74 & 441,47 & 28 \\
$1951-1989$ & 24,84 & 405,13 & 61 \\
\hline
\end{tabular}

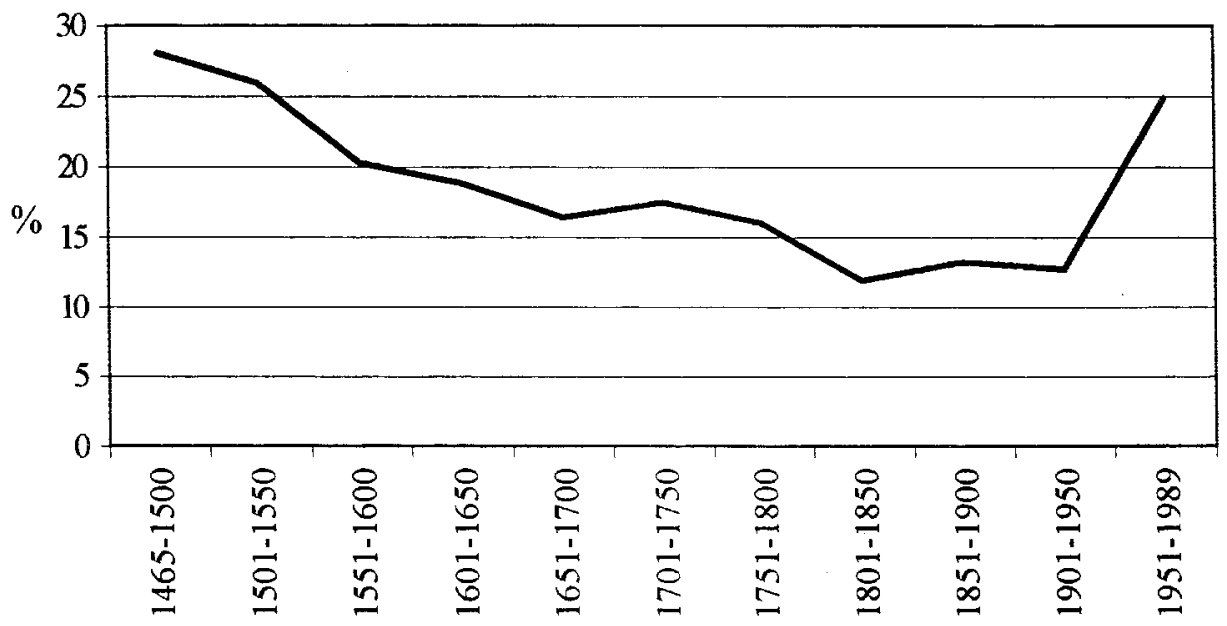

Figura 2.- Valores del coeficiente de variación de la precipitación en Pallaruelo de Monegros en intervalos de medio siglo (1465-1989). 
A finales del siglo $X V$ ofrece un valor muy elevado, que desciende de forma paulatina durante los siglos XVI y XVII, hasta mostrar un ligero repunte durante el siglo XVIII. Posteriormente se estabiliza en valores moderados para presentar en la segunda mitad del siglo $X X$ de nuevo un valor muy alto, sólo comparable al de finales del siglo XV y primera mitad del XVI.

Las rachas secas más importantes que en los apartados anteriores se han descrito, bien sea por su excepcional longitud o por mostrar unos elevados déficit medios, coinciden en el tiempo con estos momentos de mayor variabilidad pluviométrica.

El valor más alto del coeficiente de variación es del $28 \%$, y corresponde a 1 periodo 1465-1500, en el que se inscribe la racha seca más intensa de la serie y segunda en cuanto a su duración: 1488-1496. La racha seca más persistente detectada, entre 1980 y 1989, aparece en otro momento destacado por su gran variabilidad, como es 1 a segunda mitad del siglo XX. Otro periodo que registró un elevado valor del coeficiente de variación es la primera mitad del siglo XVI, en el que se han detectado tres rachas secas especialmente intensas. El siglo XVIII marca un ligero incremento del valor del coeficiente de variación que coincide con un aumento en el número e intensidad de las rachas secas (ver Tabla 3 ).

El siglo XIX y la primera mitad del siglo XX ofrecen los valores más bajos del coeficiente de variación. La presencia de años clasificados dentro de la categoría de secos sigue siendo habitual, si bien sólo se contabiliza una racha seca de duración superior a los dos años (1822-1825) y con un déficit medio moderado. Este periodo de un siglo y medio de duración caracterizado por una menor irregularidad, coincide con una menor presencia de rachas secas, mientras que las húmedas son más habituales, contabilizándose hasta siete de duración igual o mayor a los tres años. Esta situación de bonanza pluviométrica, con un importante número de años húmedos, se rompe bruscamente a mediados del siglo XX.

d) Comportamiento excepcional de los totales pluviométricos en la segunda mitad del siglo $X X$.

Varios son los trabajos que han destacado la excepcional variabilidad climática del siglo XX (respecto a los 2-3 siglos anteriores), especialmente por el elevado número de años con valores extremos. Tal frecuencia es inferior a la registrada en los siglos XV y XVI, pero entre los años 1960 y 1990 de nuevo alcanza valores que se aproximan y recuerdan a los de esas dos centurias, momento donde se ha situado la fase de mayor incidencia de la Pequeña Edad del Hielo en el ámbito de la Península Ibérica (CREUS et al., 1996). 
Ya se ha indicado que la primera mitad del siglo $X X$ junto al siglo $X I X$, conforman un periodo de menor variabilidad climática y menos presencia de secuencias secas. Por el contrario, la segunda mitad del siglo XX ofrece una imagen totalmente opuesta. El coeficiente de variación alcanza valores sólo comparables a los del siglo XV y primera mitad del XVI, además de que el porcentaje de años secos aumenta considerablemente: hasta un $61 \%$ de los años aparecen dentro de esa categoría, frente a sólo un $28 \%$ en la primera mitad del siglo.

Abundando en lo descrito cabe destacar que durante la segunda mitad de la presente centuria tiene lugar la racha seca más persistente y una de las más intensas de cuantas han sido detectadas en los últimos 525 años, la acaecida entre 1980 y 1989.

\section{CONCLUSIONES}

La aparición de rachas secas y húmedas es un fenómeno habitual en el territorio español dada su situación en la zona de contacto entre los climas templados y el dominio de las altas presiones subtropicales. Según domine una u otra influencia los años devienen más secos o húmedos, y su encadenamiento genera prolongadas sequías que van más allá de la normal reducción pluviométrica estival. En el centro de la depresión del Ebro, tales procesos se intensifican debido al aislamiento que generan los relieves circundantes (Pirineos, Sistema Ibérico y Costero Catalana) que actúan como pantalla pluviométrica frente a las perturbaciones que penetran en la Península Ibérica desde el Atlántico o el Mediterráneo. Con esta situación los periodos secos llegan a formar parte de la normalidad climática; tanto es así que los excepcional es la presencia de secuencias de años caracterizados como normales.

La racha seca más persistente acaeció entre 1980 y 1989, con una longitud de diez años. Para encontrar otra racha seca de parecida extensión hemos de remontarnos a l intervalo 1488-1496.

Las rachas húmedas suelen tener menor duración, como corresponde a una zona de gran indigencia pluviométrica. La más larga se prolongó durante ocho años (16071614), coincidiendo con la fase fría-lluviosa de la Pequeña Edad del Hielo.

El número de rachas secas de elevada intensidad es mayor que el de húmedas, siendo además las rachas secas más largas especialmente intensas. Esta relación intensidad-persistencia no aparece de forma tan clara entre las rachas húmedas.

El incremento de la variabilidad pluviométrica y la presencia de las rachas secas más importantes muestran una extraordinaria coincidencia temporal. Esta 
relación es especialmente importante en la segunda mitad del siglo $X X$ por lo que supone de ruptura con la bonanza pluviométrica y los bajos niveles de variabilidad observados en los ciento cincuenta años anteriores, y muy especialmente en la primera mitad del siglo $X X$.

\section{BIBLIOGRAFÍA}

ALBENTOSA, L.M. (1975): “La aplicación del método estadístico en climatología: 105 años de lluvias en Barcelona (1866-1970)". Revista de Geografía, vol IX.

ASCASO, A. y CASALS, M. (1981): "Periodos secos y sequías en la depresión central del Ebro". Geographicalia, 11-12, 55-71.

ALMARZA, C. y LÓPEZ, J. A. (1994): "Rachas húmedas y rachas secas durante el periodo instrumental de observaciones. El caso de San Fernando (Cádiz)", En PITA LÓPEZ, M.F. y AGUILAR ALBA, M. (Eds.): Cambios y variaciones climáticas en España. Universidad de Sevilla. Sevilla, 147-167.

CREUS, J. (1991): "Tendencia secular de la temperatura de mayo en el Pirineo Central (13021989)". Cuadernos de Geografía Física, 20-21, 41-49.

CREUS, J. (1996): "Variaciones en la disponibilidad hídrica mensual en el valle del Ebro", En MARZOL, M.V., DORTA, P. VALLADARES, P. (Eds.): Clima y agua: la gestión de un recurso climático. Universidad de La Laguna. La Laguna, 79-86.

CREUS, J., FERNÁNDEZ, A. y MANRIQUE, E. (1996): "Evolución de la temperatura y precipitación anuales desde el año 1400 en el sector central de la depresión del Ebro". Lucas Mallada, 8, 9-27

CREUS, J., FERNÁNDEZ, A. y MANRIQUE, E. (1997): "Dendrocronología y clima del último milenio en España. Aspectos metodológicos y avance de resultados". En IBÁÑEZ, J.J., VALERO, B.L., MACHADO, C. (Eds.): El paisaje mediterráneo a través del espacio y del tiempo. Implicaciones en la desertificación. Geoforma Ediciones, Logroño, 311-330.

CREUS, J. y FERRAZ, J. (1995): “Irregularidad pluviométrica y continentalidad térmica en el valle medio del Ebro". Lucas Mallada, 7, 147-164.

CREUS, J. y PUIGDEFÁBREGAS, J. (1976): “Climatología histórica y dendroclimatología de Pinus uncinata R". Cuadernos de investigación, 2 (2), 17-30.

CUADRAT, J. (1989): "Las oscilaciones climáticas recientes en Zaragoza (1865-1984)". Geographicalia, 26, 53-60.

CUADRAT, J. (1991): "La sequía en el valle del Ebro. Aspectos climáticos y consecuencias económicas". Revista Real Academia de Ciencias, 85, 537-545.

DEL VALLE, J. (1996): El clima del Prepirineo Central y Occidental aragonés y sus Somontanos. Consejo de Protección de la Naturaleza. DGA. Zaragoza. 327 pp. 
GRISOLLET, H., GUILMET, B., ARLERY, R. (1962): Climatologie. Méthodes et practiques. Gauthier-Villars, París.

PÉREZ, J. y CREUS, J. (1994):“Tendencia secular de la precipitación en Zaragoza (1865-1984)”. En PITA LÓPEZ, M.F. y AGUILAR ALBA, M. (Eds.): Cambios y variaciones climáticas en España. Universidad de Sevilla. Sevilla, 169-182. 